\title{
WAT3R: Recovery of T-Cell Receptor Variable Regions From 3' Single-Cell RNA-Sequencing
}

Marina Ainciburu ${ }^{1-3}$, Duncan M. Morgan ${ }^{4,5}$, Erica A. K. DePasquale ${ }^{2,3}$, J. Christopher Love ${ }^{3-5}$, Felipe Prósper ${ }^{1}$, Peter van Galen ${ }^{2,3}$

1. Program of Hemato-Oncology, University of Navarra, Pamplona, 31008, Spain

2. Division of Hematology, Brigham and Women's Hospital, Boston, MA, 02115, USA

3. Broad Institute of MIT and Harvard, Cambridge, MA, 02142, USA

4. Department of Chemical Engineering, Massachusetts Institute of Technology, Cambridge, MA, 02139, USA

5. Koch Institute for Integrative Cancer Research, Massachusetts Institute of Technology, Cambridge, MA, 02139, USA

\section{Correspondence:}

Peter van Galen, PhD

Assistant Professor of Medicine, Harvard Medical School

Hematology Division, Brigham and Women's Hospital

4 Blackfan Circle, Harvard Institutes of Medicine (HIM) 741, Boston MA 02115

Email: petervangalen@bwh.harvard.edu 


\section{Abstract}

\section{Summary}

Diversity of the T-cell receptor (TCR) repertoire is central to adaptive immunity. The TCR is composed of $\alpha$ and $\beta$ chains, encoded by the TRA and TRB genes, of which the variable regions determine antigen specificity. To generate novel biological insights into the complex functioning of immune cells, combined capture of variable regions and single-cell transcriptomes provides a compelling approach. Recent developments enable the enrichment of TRA and TRB variable regions from widely used technologies for 3'-biased single-cell RNA-sequencing (scRNA-seq). However, a comprehensive computational pipeline to process TCR-enriched data from 3' scRNA-seq is not available. Here we present an analysis pipeline to process TCR variable regions enriched from 3' scRNA-seq cDNA. The tool reports TRA and TRB nucleotide and amino acid sequences linked to cell barcodes, enabling the reconstruction of T-cell clonotypes with associated transcriptomes. We demonstrate the software using peripheral blood mononuclear cells (PBMCs) from a healthy donor and detect TCR sequences in a high proportion of single T-cells. Detection of TCR sequences is negligible in non-T-cell populations, demonstrating specificity. Finally, we show that TCR clones are larger in CD8 Memory T-cells than other T-cell types, indicating an association between T-cell clonotypes and differentiation states.

\section{Availability and implementation}

The Workflow for Association of T-cell receptors from 3' single-cell RNA-seq (WAT3R), including test data, is available on GitHub (https://github.com/mainciburu/WAT3R), Docker Hub (https://hub.docker.com/r/mainciburu/wat3r), and a workflow on the Terra platform (https://app.terra.bio). The test dataset is available on GEO (accession number pending).

\section{Introduction}

During T-cell development, a series of recombinations shape the $\alpha$ and $\beta$ chains that comprise the TCR, giving rise to $10^{15}-10^{21}$ potential TCRs (La Gruta et al., 2018). The recombinations occur in the variable regions of the TRA and TRB genes that encode the TCR $\alpha / \beta$ chains. TRA and TRB determine T-cell specificity by shaping TCR recognition of antigens presented by $\mathrm{MHC}$ molecules. The recombined sequences can also be used to track T-cell clonotypes. With the advent of single-cell sequencing technologies, simultaneous capture of TRA and TRB sequences combined with transcriptional states provides a powerful approach to study T-cell biology (Stubbington et al., 2016).

Several protocols have been developed to combine scRNA-seq with recovery of TCR sequences, using cell barcodes to integrate both layers of information. Low-throughput single-cell methods performed on multiwell plates allow for the recovery of the complete TRA and TRB genes (Sade-Feldman et al., 2018; Stubbington et al., 2016). On the other hand, high-throughput methods based on microfluidics or microwell chips create libraries that can be enriched for TCR sequences (Oliveira et al., 2021; Zemmour et al., 2018; Oh et al., 2020). Among the latter, T-cell Receptor Enrichment to linK clonotypes (TREK-seq) is a recently published protocol to perform both transcript and TCR sequencing from 10x Genomics 3' Gene Expression libraries (Miller et al., 2022; Tu et al., 2019). Although analysis tools exist for TCR bulk analysis (Bolotin et al., 2015) and 5' single-cell protocols 
(CellRanger), there is a need for bioinformatics tools that facilitate the analysis of TCR variable regions enriched from 3' scRNA-seq cDNA. Here, we describe WAT3R (pronounced "water"), an integrated pipeline that covers TCR-enriched data from preprocessing FASTQ files to alignment and the identification of T-cell clones.

\section{Description}

In accordance with the TREK-seq protocol, sequencing data is provided as two compressed FASTQ files. One contains the cell barcode and unique molecular identifier (UMI) sequences and the other the TCR sequence. First, files are reformatted to join the barcode, UMI, and corresponding TCR sequence in a single FASTQ. Next, the user can specify whether barcode and UMI correction should be performed. Barcode correction allows for one mismatch with barcodes in the 10x Single Cell 3' v3 list (or a custom list provided by the user). UMI correction is performed by clustering together UMIs with one mismatch and considering the most abundant UMI as the correct one. Every barcode and UMI sequence is then added to the corresponding FASTQ read header, to be used as an identifier. Next, we apply a quality filter to remove every read with an average quality score lower than indicated (default is qscore $<25$, Figure 1, top left). To account for barcode swapping (i.e., incorrect barcode assigning), TCR sequences with an identical barcode and UMI are subjected to clustering based on sequence similarity, using the USEARCH algorithm (Edgar, 2010). The identity threshold to measure similarity can be set by the user (default is 0.9 ). To ensure high data consistency, only TCR sequences are kept if the most abundant cluster represents a large proportion of the reads (default is 0.5 ) and is substantially larger than the second most abundant cluster (default ratio is 2.0) (Figure 1, top right).

Next, a consensus sequence is built for each of the clusters (Heiden et al., 2014). For a consensus to be constructed, we require a minimum of 3 reads and allow for a maximum error rate of 0.5 and a gap frequency of 0.5 per position; these parameters can be changed by the user (Figure 1, bottom left). Consensus sequences are aligned to the $V(D) J$ segments reference provided by IMGT, using IgBLAST with the default parameters (Ye et al., 2013; Lefranc et al., 2015). This task is performed through the interface implemented in the Python package Change-O (Gupta et al., 2015). After selecting TRA and TRB sequences with the highest UMI counts, the CDR3 nucleotide/amino acid sequences and $V(D) J$ calls are assigned to cell barcodes and saved in a results table.

As an optional step, the user can provide a file with cell barcodes and annotations coming from a paired scRNA-seq experiment to integrate with the TRA and TRB calls (Figure 1, bottom right). Overall, this pipeline returns two tables of results, one at the transcript level and another at the cell level. In addition, multiple quality control (QC) graphs and metrics are generated. WAT3R, together with the required software, reference data and documentation is available as a docker image. It is also available as a workflow on Terra which provides access to Google Cloud computing resources through a simple web-based user interface.

\section{Results}

We analyzed a human peripheral blood sample using 10x Genomics 3' v3 scRNA-seq and TREK-seq to enrich TRA and TRB variable regions (Miller et al., 2022). The TREK-seq 


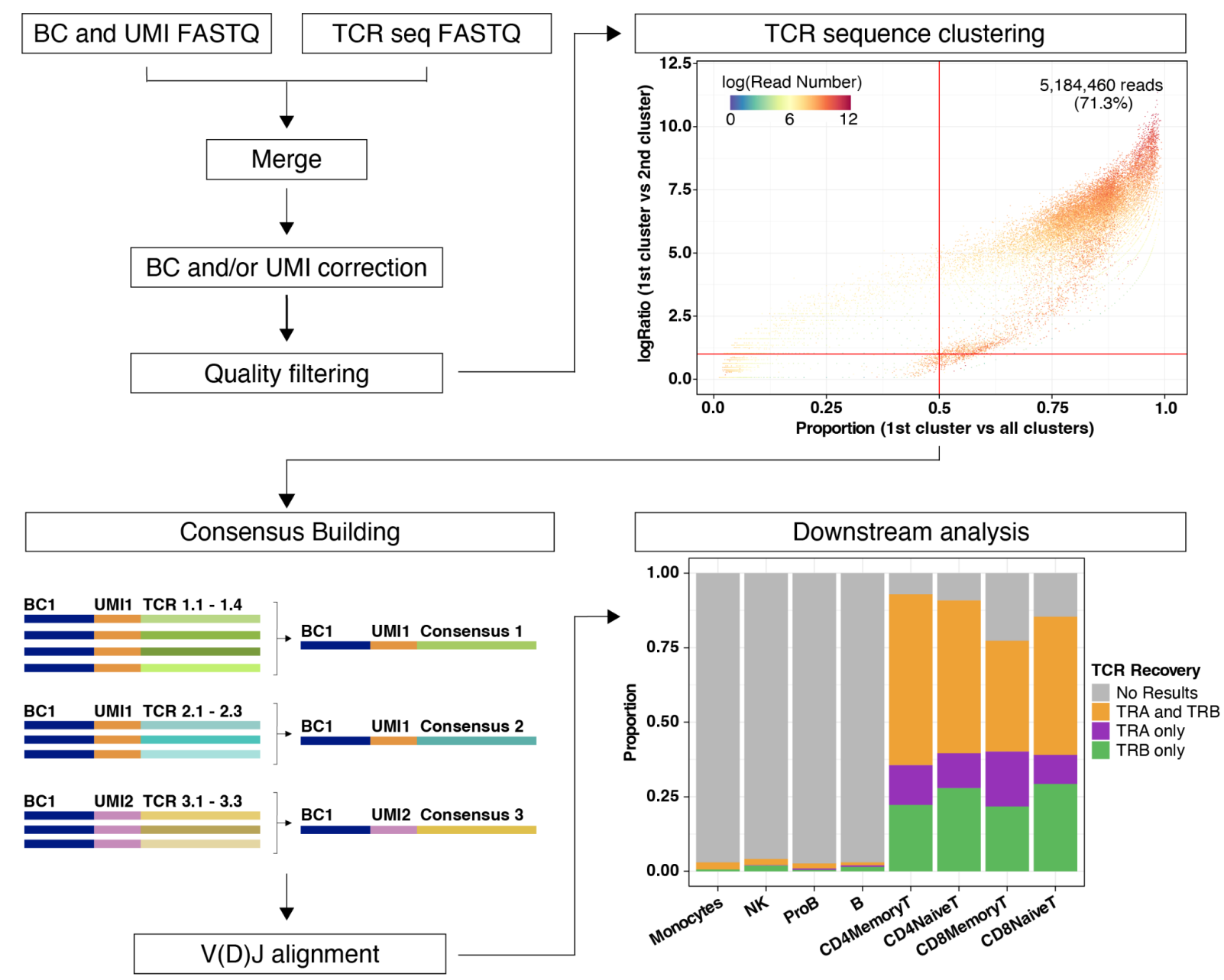

Figure 1. Overview of WAT3R. The workflow starts by merging two FASTQ files, correction of cell barcodes and UMIs, and quality filtering (top left). Clustering of TCR sequences with identical barcode and $\mathrm{UMI}$ is then performed. Top right dotplot shows evaluation of cluster quality by comparing the proportion of reads supporting the most abundant cluster ( $x$-axis), the ratio of the most abundant cluster to the second (y-axis), and the number of reads supporting each TCR sequence with a specific barcode and UMI (color). Bottom left: TCR consensus sequences are generated and used for V(D)J alignment. In downstream analysis, results are integrated with a paired ScRNA-seq dataset. Bottom right barplot shows the proportion of cells in the dataset, separated by cell type, for which WAT3R returned information on the TRA gene, TRB gene, or both.

library was sequenced on a MiSeq to a depth of 8 million reads. After recovering $4.3 \%$ of the cell barcodes using the barcode correction algorithm, $98.3 \%$ of reads contained valid barcodes (Supplementary Figure 1). Likewise, $4.9 \%$ of the UMI sequences were corrected. Reads were filtered for an average q score above 25 , which retained $92.3 \%$ of the original reads. For consensus building and subsequent alignment, $70.4 \%$ of the reads were valid. After removal of TCR sequence clusters below the proportion and ratio thresholds, $65.8 \%$ of the original reads were retained. These reads were used to generate a results table (Supplementary Figure 2).

We integrated these results with the paired 3' scRNA-seq dataset with cell type annotations based on canonical marker genes (Supplementary Figure 3) (Hao et al., 2021). Detection of TRA or TRB sequences in $90 \%$ of all T-cells demonstrates efficient enrichment from the 
cDNA. In contrast, TRA or TRB sequences are present in only $3 \%$ of non-T-cells, demonstrating specificity. The TRB variable region is most efficiently enriched: TRA sequences are detected in $65 \%$ of single T-cells, TRB in $77 \%$, and TRA+TRB in $52 \%$ (Figure 1 , bottom right). As expected in a healthy individual, we did not observe any expanded T-cell clones dominating the sample. Nonetheless, the largest detected clones belong mainly to CD8 Memory T-cell subsets, in accordance with previous findings (Supplementary Figure 4) (DePasquale et al., 2021; Penter et al., 2021).

\section{Conclusions}

WAT3R is a comprehensive pipeline for the analysis of TRA and TRB variable regions enriched from the cDNA of widely used 3' scRNA-seq protocols. From sequencing error correction, alignment, and quality controls to intersection with cell type annotations from scRNA-seq, this tool applies state-of-the-art algorithms to reliably detect T-cell clonotypes and initiate new discoveries in immunology.

\section{Acknowledgments}

We thank the healthy donor for donating peripheral blood cells. We thank Julia Verga, Tyler Miller, Martin Villanueva, Charles Couturier, Daniel Ssozi, Jonathan Good, Jenny Noel and Alex Shalek for help with the TREK-seq protocol development and Yoke Seng Lee and Antonia Kreso for helpful feedback.

\section{Funding}

P.v.G. is supported by the Ludwig Center at Harvard, the NIH (R00CA218832), Gilead Sciences, the Bertarelli Rare Cancers Fund, the William Guy Forbeck Research Foundation, and is an awardee of the Glenn Foundation for Medical Research and American Federation for Aging Research (AFAR) Grant for Junior Faculty. M.A. is supported by a PhD fellowship (FPU18/05488) and a mobility scholarship from the Government of Spain.

Conflict of interest: none declared.

\section{References}

Bolotin,D.A. et al. (2015) MiXCR: software for comprehensive adaptive immunity profiling. Nat. Methods, 12, 380-381.

DePasquale,E.A.K. et al. (2021) Single-cell Multiomics Reveals Clonal T-cell Expansions and Exhaustion in Blastic Plasmacytoid Dendritic Cell Neoplasm. bioRxiv, 2021.12.01.470599.

Edgar,R.C. (2010) Search and clustering orders of magnitude faster than BLAST. Bioinformatics, 26, 2460-2461.

Gupta,N.T. et al. (2015) Change-O: a toolkit for analyzing large-scale B cell immunoglobulin repertoire sequencing data. Bioinformatics, 31, 3356-3358.

Hao,Y. et al. (2021) Integrated analysis of multimodal single-cell data. Cell, 184, 3573-3587.e29.

Heiden,J.A.V. et al. (2014) pRESTO: a toolkit for processing high-throughput sequencing raw reads of lymphocyte receptor repertoires. Bioinformatics, 30, 1930-1932. 
La Gruta,N.L. et al. (2018) Understanding the drivers of MHC restriction of T cell receptors. Nat. Rev. Immunol., 18, 467-478.

Lefranc,M.-P. et al. (2015) IMGT®, the international ImMunoGeneTics information system® 25 years on. Nucleic Acids Res., 43, D413-22.

Miller,T.E. et al. (2022) Mitochondrial variant enrichment from high-throughput single-cell RNA-seq resolves clonal populations. Nat. Biotechnol.

Oh,D.Y. et al. (2020) Intratumoral CD4+ T Cells Mediate Anti-tumor Cytotoxicity in Human Bladder Cancer. Cell, 181, 1612-1625.e13.

Oliveira,G. et al. (2021) Phenotype, specificity and avidity of antitumour CD8+ T cells in melanoma. Nature, 596, 119-125.

Penter,L. et al. (2021) Coevolving JAK2V617F+ relapsed AML and donor T cells with PD-1 blockade after stem cell transplantation: an index case. Blood Adv.

Sade-Feldman,M. et al. (2018) Defining T Cell States Associated with Response to Checkpoint Immunotherapy in Melanoma. Cell, 175, 998-1013.e20.

Stubbington,M.J.T. et al. (2016) T cell fate and clonality inference from single-cell transcriptomes. Nat. Methods, 13, 329-332.

Tu,A.A. et al. (2019) TCR sequencing paired with massively parallel 3' RNA-seq reveals clonotypic T cell signatures. Nat. Immunol., 20, 1692-1699.

Ye,J. et al. (2013) IgBLAST: an immunoglobulin variable domain sequence analysis tool. Nucleic Acids Res., 41, W34-40.

Zemmour,D. et al. (2018) Single-cell gene expression reveals a landscape of regulatory $T$ cell phenotypes shaped by the TCR. Nat. Immunol., 19, 291-301. 


\section{Supplementary Data}

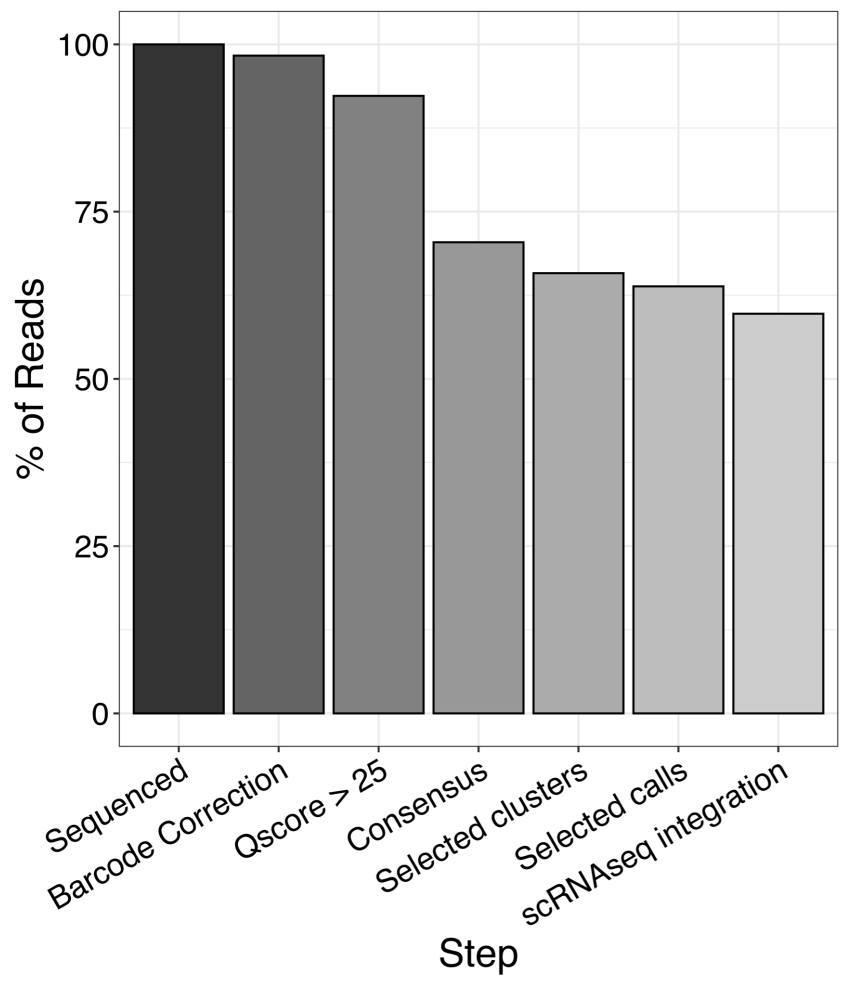

Supplementary Figure 1. Retainment of raw sequencing reads. Barplot shows the percentage of reads passing the different filtering steps throughout the pipeline. 


\begin{tabular}{|c|c|c|c|c|c|}
\hline BC & TCR_Recovery & TRB_CDR3 & $\begin{array}{l}\text { TRB_CDR3_ } \\
\text { UMlcount }\end{array}$ & TRB_nReads & RNAannotation \\
\hline CCTCAGTAGTGCGACA & TRA and TRB & CASSIGQGAHDGYTF & 27 & 12728 & 3 CD4MemoryT \\
\hline GCTGCAGCACGCGCTA & TRA and TRB & CASSPTPGRNYGYTF & 33 & 9067 & 7 CD4MemoryT \\
\hline CTGTACCAGGAATCGC & TRA and TRB & CASSLYGTGELFF & 17 & 5984 & CD4MemoryT \\
\hline ATCGTGATCATTCGGA & TRA and TRB & CASSRLTDRDEQFF & 24 & 5973 & 3 CD4MemoryT \\
\hline AAACGAATCCGCACTT & TRA and TRB & CASSYGTGGYQPQHF & 16 & 5344 & CD4MemoryT \\
\hline ATCACTTCACTTCTCG & TRA and TRB & RASSLYLRARAWDSNQPQHF & 15 & 5223 & 3 CD4NaiveT \\
\hline TTCAGGATCAGACATC & TRA and TRB & CASSSGTGATTEAFF & 16 & 5065 & CD4NaiveT \\
\hline CTTTCAAAGAATTGCA & TRA and TRB & CASRETGTGHNTEAFF & 12 & 5061 & CD4NaiveT \\
\hline GCTTCACTCGCGTTTC & TRA and TRB & CASSRSGAIRETQYF & 19 & 4234 & CD4MemoryT \\
\hline AGACAAAGTAGAGGAA & TRA and TRB & CASSLVQGSDKTYEQYF & 11 & 4153 & CD4MemoryT \\
\hline TCCATGCTCCTCGATC & TRA and TRB & CASSDSTPNTGELFF & 15 & 4038 & 3 CD4NaiveT \\
\hline TCСАССАТСАТTСАСТ & TRA and TRB & CSAKKELGFTDTQYF & 12 & 3857 & 7 CD4MemoryT \\
\hline GGTTGTACAACAGAGC & TRA and TRB & CASSPGQGGQPQHF & 11 & 3846 & 5 CD4NaiveT \\
\hline ATTTCTGTCTTACCAT & TRA and TRB & CASSPGGGTGELFF & 17 & 3828 & 3 CD4MemoryT \\
\hline CGGACACAGACGTCCC & TRA and TRB & CASSRTGVEAFF & 10 & 3823 & 3 CD4MemoryT \\
\hline GGCTTTCTCCCGATCT & TRA and TRB & CASSKISGGYAGEQFF & 9 & 3697 & CD4NaiveT \\
\hline GACGCTGCACAATCTG & TRA and TRB & CASSLAGQETQYF & 11 & 3621 & CD4MemoryT \\
\hline AGGTGTTGTTAGGGAC & TRB only & CASVGLDTQYF & 10 & 3615 & CD4NaiveT \\
\hline TCATGTTTCCACACCT & TRA and TRB & CASSSSGGAHNEQFF & 14 & 3586 & 5 CD4MemoryT \\
\hline TAACGACCAAAGGTTA & TRA and TRB & CASSRQENQPQHF & 10 & 3563 & 3 CD4MemoryT \\
\hline AACAAGACATCTATCT & TRB only & CASSLNPGLSGANVLTF & 8 & 3551 & CD4MemoryT \\
\hline CATTTCAGTGGCTTAT & TRA and TRB & CASSFGYQGAGYGYTF & 11 & 3514 & CD4NaiveT \\
\hline AATGAAGAGTAGAGTT & TRA and TRB & CASSLATLGTDTQYF & 13 & 3445 & CD4MemoryT \\
\hline ATAGACCCACTCTAGA & TRB only & CASRQSPTYEQYF & 11 & 3432 & CD4MemoryT \\
\hline GGGTGTCGTCGAACGA & TRB only & CASSQDLTRPPQHF & 10 & 3409 & CD4NaiveT \\
\hline
\end{tabular}

Supplementary Figure 2. Excerpt of the results table. The complete results table contains additional columns and rows, including CDR3 loop nucleotide sequences, predicted $\mathrm{V}(\mathrm{D}) \mathrm{J}$ segments and TRAV calls. BC: cell barcode, TCR_Recovery: TRA, TRB or both, TRB_CDR3: predicted amino acid sequence of the CDR3 loop, TRB_CDR3_UMlcount: number of UMls (transcripts) supporting the TRB call, TRB_nReads: number of reads supporting the TRB call, RNAannotation: cell type annotation from scRNA-seq. 


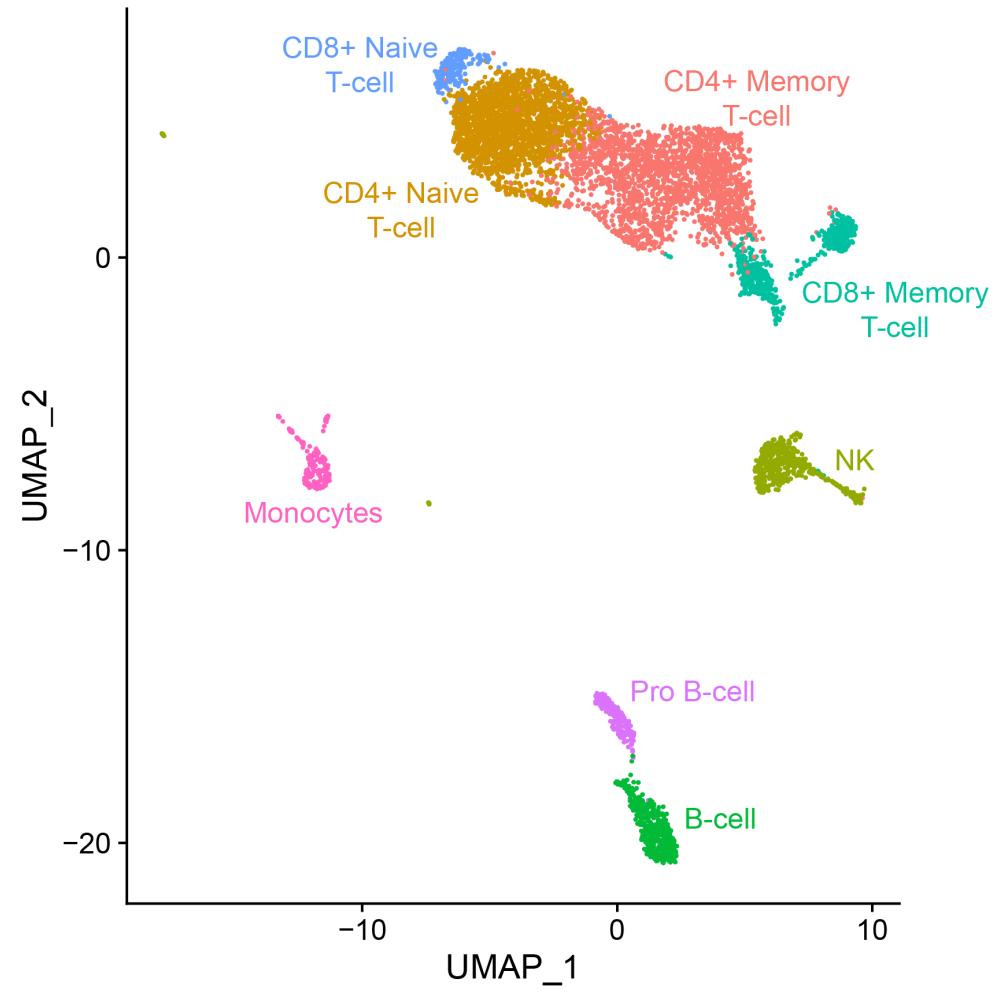

Supplementary Figure 3. UMAP with cell type annotations. UMAP shows 6,300 cells from a healthy PBMC donor along with cell type annotations. Dimensionality reduction and clustering was performed using standard procedures (Hao et al., 2021). The cell type annotations correspond to the bottom right panel of Figure 1.

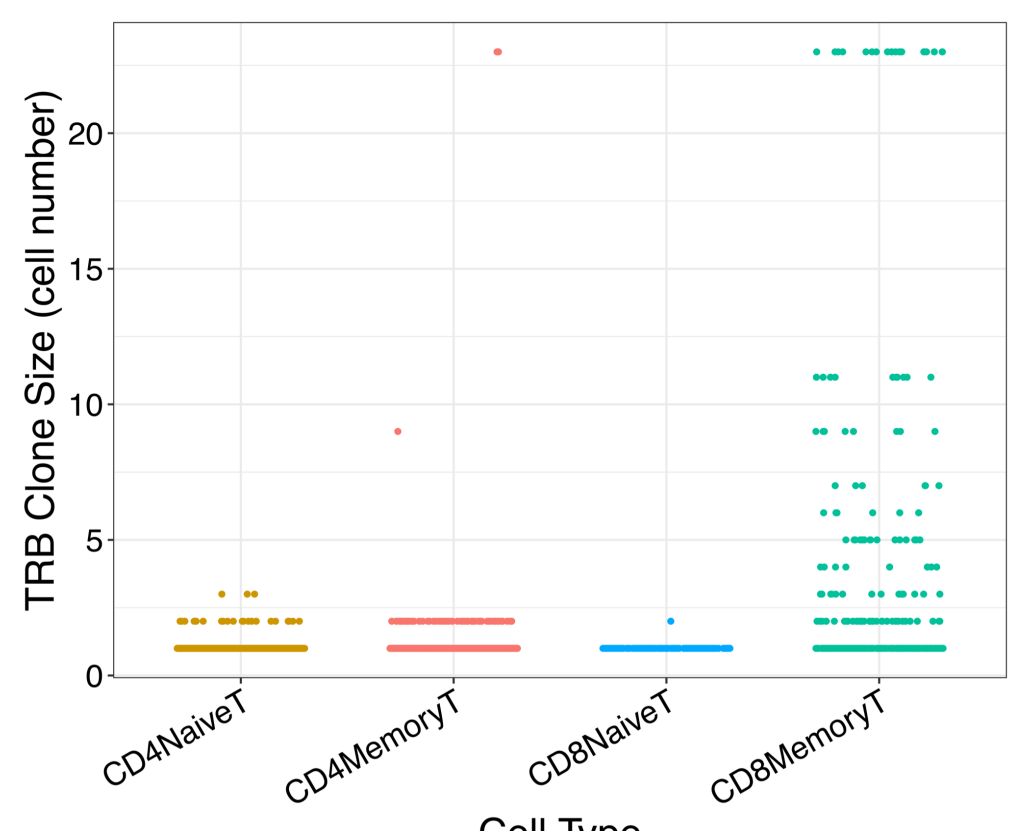

Cell Type

Supplementary Figure 4. T-cell clonotype sizes are largest in CD8 Memory T-cells. Dot plot shows T-cell clone sizes (number of cells with the same TRB sequence) in four different T-cell populations from the healthy PBMC donor. Clone sizes are significantly larger in CD8 Memory T-cells compared to any of the other populations $(P<0.05)$. 